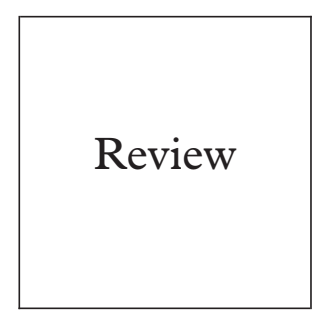

\title{
AIDS related systemic non-Hodgkin's lymphoma
}

\author{
Tom Powles, Gail Matthews, Mark Bower
}

Objective: To review the current literature on HIV associated non-Hodgkin's lymphoma.

Methods: A comprehensive Medline/Pubmed search of articles pertaining to HIV associated non-Hodgkin's lymphoma as well as personal experience from the treatment of over 200 patients at the Chelsea and Westminster Hospital, one of the largest centres for the management of HIV disease in Europe.

Conclusion: High grade B cell non-Hodgkin's lymphoma is the second commonest tumour affecting people with HIV. The incidence of this tumour is not declining following the introduction of highly active antiretroviral therapy. Chemotherapy has been employed with modest success in this group of patients; however, the prognosis remains worse than for immunocompetent patients. Advances in molecular genetics and virology have led to a greater understanding of the biology of these tumours. However, these advances have yet to be translated into improvements in the clinical management of patients with AIDS associated non-Hodgkin's lymphoma.

(Sex Transm Inf 2000;76:335-341)

Keywords: AIDS; HIV; non-Hodgkin's lymphoma

\section{Introduction}

Since 1981 and the emergence of the HIV pandemic, a close association between HIV infection and the development of a selected group of cancers has been noted. These malignancies include Kaposi' sarcoma, high grade B cell non-Hodgkin's lymphoma, anal cancer, and invasive cervical cancer. Most of these tumours have also been found to occur at a high incidence in patients with other forms of immunosuppression including iatrogenic immunosuppression following allograft transplantation. ${ }^{1}$ It has emerged that viral oncogenesis may have a central role in the development of all of these tumours and that this could be the reason why these cancers are found more frequently in immunocompromised patients. Interestingly, hepatocellular carcinoma does not occur more often in HIV positive patients, although coinfection with HIV and hepatitis B and C is common. ${ }^{2}$

This article discusses the features of high grade B cell non-Hodgkin's lymphoma in the setting of HIV and reviews the changes in the disease and treatment as the AIDS pandemic has developed.

\section{Epidemiology}

High grade B cell non-Hodgkin's lymphoma (NHL) has been classified as an AIDS defining illness since 1985 following the description of NHL in 90 HIV seropositive men. ${ }^{3}$ In the era that preceded the introduction of highly active antiretroviral therapy (HAART) in 1996, NHL was diagnosed approximately 60 times more often in the HIV positive population than in the general population. ${ }^{4}$ Among this group it appears to be slightly commoner in haemophiliacs and less common in injecting drug users. ${ }^{4}$ In the pre-HAART era the incidence of AIDS related lymphoma remained fairly constant at around six to seven cases per 1000 person years. ${ }^{5}$ NHL may be the AIDS defining ill- ness or may occur in people who already have an AIDS defining diagnosis. NHL accounted for only $1.5 \%$ of first AIDS defining illnesses, ${ }^{5}$ but was estimated to be $3.0-3.6 \%$ of all AIDS defining illness each year before $1996 .{ }^{67}$ Since the development of NHL is associated with a relative risk of death of 20 -fold, ${ }^{8}$ HIV associated NHL caused up to $16 \%$ of all deaths attributable to AIDS. ${ }^{49}$

The widespread introduction of HAART since 1996 has led to dramatic reductions in AIDS related morbidity and mortality throughout the developed world..$^{11}{ }^{11}$ This is largely due to the marked fall in incidence of the major opportunistic infections such as Pneumocystis carinii pneumonia (PCP), cytomegalovirus (CMV), and Mycobacterium avium complex (MAC). Although this decline has also been observed in Kaposi's sarcoma, the effect on NHL has not been so obvious. In a Swiss HIV cohort study, a substantial relative risk reduction from 1992-4 to 1997-8 was seen for development of Kaposi's sarcoma as well as other AIDS defining conditions but no significant trend was seen for NHL, implying a continuing risk for patients despite the use of potent antiretroviral therapies. ${ }^{12}$ Other groups from the United States confirm this finding. The US AIDS Clinical Trial Group found the incidence of both Kaposi's sarcoma and NHL to be decreasing but the former far more profoundly and consistently. ${ }^{13}$ A group from the San Francisco City Clinic found the incidence of lymphoma between 1993 and 1996 to be unchanged at $1.4-1.8$ per 100 person years. ${ }^{14}$ The Multicenter AIDS Cohort Study (MACS) actually found an increase in the incidence of NHL at a rate of $21 \%$ per year ${ }^{15}$ while the incidence of Kaposi's sarcoma fell by $66 \%$ during the same period.

As a percentage of AIDS defining illnesses NHL has increased; in Western Europe it rose from $3.6 \%$ in 1994 to $4.9 \%$ in $1997 .^{7}$ This, 
however, is likely to reflect the reduction in other causes of AIDS more than a true rise in incidence. It may be that with increasing use and duration of HAART both the incidence and characteristics of AIDS associated lymphoma will change. NHL may not be falling despite HAART because HAART may not prevent chronic HIV related $B$ cell stimulation, and other aetiological factors involved with the pathogenesis of NHL may be unaffected by HAART.

\section{Histopathology}

The classification of NHL aims to help the pathologist to recognise the diagnosis, the clinician to treat the patient, and the scientist to unravel the biology of the tumour. Initial classifications, such as the Rappaport classification, were based upon the morphological appearance of formalin fixed, haematoxylin and eosin stained tumours. Subsequent classifications have incorporated the use of immunophenotyping of cluster designation (CD) antigens, cytogenetics, and molecular analysis. The myriad of classifications that have evolved as a consequence of these improvements have rarely helped the pathologist or clinician and mainly confused the biologist. The latest, but certainly not last, clarification of NHL is the Revised European and American Lymphoma classification (REAL). Within this classification approximately two thirds of all AIDS related NHL are diffuse large cell lymphomas (which includes all immunoblastic lymphomas) while one third are small non-cleaved cell lymphomas (which includes Burkitt's lymphomas). ${ }^{316-21}$

Primary effusion lymphoma (PEL) or body cavity based lymphoma (BCBL) is a rare variant of HIV associated lymphoma that is characterised by effusions in serosal cavities (pleura, pericardium, peritoneum) in the absence of solid nodal masses. PEL are CD30 (Ki-1) positive anaplastic large cell lymphomas which express an indeterminate immunophenotype with clonal immunoglobulin gene rearrangements. All PEL are associated with human herpes virus 8 (HHV8) infection and tumour cells carry a high HHV8 viral copy number. ${ }^{22}$ In addition, many PEL are coinfected with Epstein-Barr virus (EBV) making the relative roles of the two herpesviruses in the pathogenesis of PEL difficult to determine. Certainly, HHV8 expresses a limited repertoire of latent phase genes in PEL cells and these could play a part in cellular transformation. The clinical management of PEL does not differ from the HIV associated NHL, although patients frequently have advanced immunosuppression at diagnosis. Patients present with a median CD 4 count of $90 \times 10^{6} / 1$ while the median survival is 5 months.

Castleman's disease is a rare lymphoproliferative disorder originally described in 1956 and characterised by angiofollicular lymphoid hyperplasia. Two histological variants are recognised; a hyaline vascular variant and a less common plasma cell variant. Castleman's disease has germinal centre hyalinisation or atrophy surrounded by concentric layers of lymphocytes with prominent vascular hyperplasia, hyalinisation of small vessels, and interfollicular sheets of plasma cells and immunoblasts. Patients present with constitutional symptoms including fever, weight loss, and night sweats. Clinical findings include lymphadenopathy, hepatosplenomegaly, and rashes. Investigations frequently reveal microcytic anaemia, hypoalbuminaemia, and polyclonal hypergammaglobulinaemia. The optimum treatment for Castleman's disease remains uncertain. Early reports of multicentric Castleman's disease in seropositive patients suggested a median survival of less than 6 months. However, earlier recognition of the diagnosis and treatment with splenectomy followed by single agent chemotherapy may prolong survival. In the largest reported series from Paris of 20 patients treated with splenectomy and vinblastine, the median survival was 14 months. ${ }^{23}$ A recent study has demonstrated that HHV8 is specifically associated with a variant of multicentric Castleman's disease with $\lambda$ light chain restricted HHV8 positive plasmablasts in the mantle zone of B cell follicles. There is a high frequency of plasmablastic lymphoma associated with multicentric Castleman's disease and these lymphomas are also positive for HHV8 and $\lambda$ light chain. ${ }^{24}$

\section{Pathogenesis}

It is thought that immune stimulation by HIV virus $^{25}$ and reactivation of previous EBV infection due to defective $T$ cell surveillance, leads to long term stimulation and proliferation of $\mathrm{B}$ lymphocytes resulting in the development of AIDS related NHL. Moreover, even in the absence of EBV infection, HIV induces the production of inflammatory cytokines that cause $\mathrm{B}$ cell stimulation, proliferation, and activation, ${ }^{26-28}$ and cell lines derived from AIDS related NHL have been found to express cytokines including interleukin 6 , interleukin 10 , and tumour necrosis factor $\beta .^{29}$

Primary infection of epithelial cells by EBV is associated with the infection of some resting B lymphocytes via the CD21 receptor. Most infected B lymphocytes express EBV latently with a type 3 latency expression, producing up to six EBV nuclear antigens (EBNAs) as well as latent membrane proteins (LMP) 1, 2A, and 2B. Some B cells appear to switch to a latency 1 pattern expressing only EBNA-1 a nuclear antigen and these cells may escape destruction by cytotoxic $\mathrm{T}$ lymphocytes and persist. They are believed to be the origin of EBV reactivation and EBV positive NHL. Indeed, EBV genomic terminal analysis has shown clonal EBV infection in HIV associated NHL, implying that EBV infection precedes clonal expansion. HIV associated diffuse large cell and immunoblastic lymphomas are frequently associated with EBV. The EBV genome in these lymphomas expresses latency type 3 antigens including EBNA-2 and LMP-1 and 2 which have transforming activity in vitro. In addition, cellular genes are expressed including the cellular adhesion molecule ICAM-1 (intercellular adhesion molecule 1 or CD54), the integrin LFA-1 (lymphocyte function associ- 
ated antigen 1 or CD18), and the addressin LHR (ligand lymphocyte homing receptor or CD44). However, the EBV genome can be detected in only $60 \%$ of these HIV associated large cell lymphomas ${ }^{30}$ compared with almost all cases of post-transplantation NHL. This implicates other factors in the aetiology of these malignancies associated with HIV infection, including polyclonal B cell expansion and impaired $\mathrm{T}$ cell immunosurveillance. In contrast, EBV is present in around 30\% HIV associated Burkitt's lymphoma and in these tumours EBV is found in the latency type 1 profile expressing only EBNA-1 and a small abundant non-translated RNA (EBER) ${ }^{30}$ A second herpesvirus, HHV8, that is causally implicated in Kaposi's sarcoma is also implicated in a subset of AIDS related NHL. HHV8 has been demonstrated in both HIV associated primary effusion lymphoma and Castleman's disease $^{22} 31$

Burkitt's lymphoma (BL) is not associated with other forms of immunosuppression and occurs in endemic and sporadic forms. The former is a childhood lymphoma prevalent in equatorial Africa and intimately associated with both EBV and a characteristic chromosomal translocation involving $c-m y c$. The sporadic version of the disease which affects young adults is less frequently associated with EBV infection and similar but distinct genetic rearrangements are seen. The HIV associated $\mathrm{BL}$ resembles sporadic $\mathrm{BL}$ in that only $30 \%$ are EBV positive and $c-m y c /$ immunoglobulin gene translocations are found in most cases. The translocation breakpoints on chromosome 8 occur most frequently in exon 1 or intron 1 of the $c-m y c$ gene in AIDS related NHL rather than 5' upstream of the gene. This pattern of translocation break points on $c-m y c$ do not alter the peptide sequence since exon 1 is not translated, and resemble the break points observed in sporadic BL rather than endemic BL. ${ }^{32-34}$ The reciprocal break point most often found lies in the $S \mu$ portion of the immunoglobulin heavy chain gene on chromosome 14. This pattern, which is also found in sporadic BL suggests that the mechanism of translocation is defective recombination during isotype class switching of the constant region of the heavy chain of immunoglobulin, which occurs late in B lymphocyte ontogeny in the germinal centres.

In addition to EBV and $c-m y c$ other genetic events have been identified in AIDS related NHL that may contribute to the pathogenesis of these tumours. The tumour suppressor gene P53 has a central role in cell cycle control and hence regulates cell replication. About $40 \%$ of HIV associated NHL have been found to have mutations of p53 gene. These mutations are found most commonly associated with the small non-cleaved cell or Burkitt-like variants rather than the diffuse large cell histologies. In contrast, mutations of the retinoblastoma gene which is also a cell cycle regulator have not been found in HIV associated NHL.

Chromosome 3q27 translocations in diffuse large cell lymphomas led to the identification of a novel oncogene bcl-6. Bcl-6 encodes a zinc finger transcription factor which is selectively expressed in germinal centre B lymphocytes. This gene is rearranged in $30-40 \%$ diffuse large cell lymphomas and rearrangements have been found in $20 \%$ HIV associated NHL including mutations of the 5 ' non-coding regulatory sequences. ${ }^{35-37}$ In contrast, rearrangements of Bcl-1 and Bcl-2 have not been demonstrated in AIDS associated NHL.

Genetic markers may identify HIV infected people at a particularly high risk of developing HIV related NHL. Rabkin et al have studied chemokine and chemokine receptor gene variants in this context. Stromal cell derived factor 1 is a polymorphic chemokine with $37 \%$ white and $11 \%$ black Americans carrying the 3'A variant. The SDF-1 3'A variant was associated with a doubling of the risk of HIV associated NHL in heterozygotes and quadrupling of the risk in homozygotes. In contrast, the presence of the chemokine receptor variant CCR5 $\Delta 32$ is protective against the development of NHL while the CCR2-64I variant has no significant effect. $^{38}{ }^{39}$ People who carry the CCR5 $\Delta 32$ deletion do not express CCR5 and have been found to be resistant to infection by HIV despite frequent exposure. ${ }^{40-42}$ CCR5 is the macrophage coreceptor for macrophage tropic non-syncytium inducing strains of HIV. In contrast, variants of CCR2, another $\beta$ chemokine receptor, are associated with nonprogression of HIV to AIDS.

The role of HIV itself in the development of NHL appears to be confined to its lymphoproliferative effects; indeed one tumour has been described that secreted a monoclonal paraprotein directed at the gp 160 antigen of HIV. ${ }^{43}$ In rare tumours HIV may be playing a more direct part as a transforming oncogenic virus and one HIV associated T cell tumour has been shown to harbour monoclonally integrated HIV in the tumour genome. ${ }^{44}$

\section{Clinical features}

Patients with HIV associated NHL more frequently present with advanced disease and/or extranodal disease than immunocompetent patients and B symptoms occur in up to $90 \%$ of patients. ${ }^{45}{ }^{46}$ The commonest site of extranodal disease is the gastrointestinal tract $^{47}$ particularly the small intestine, stomach, and perianal region. Similarly, hepatic involvement occurs in up to one quarter of patients and results have suggested that the prognosis is particularly poor. ${ }^{48}$ Bone marrow involvement by lymphoma occurs in up to $20 \%,{ }^{49}$ although trilineage myelodysplasia is almost ubiquitous owing to the HIV and may add to the myelotoxicity of cytotoxic chemotherapy treatments. In addition to primary cerebral lymphoma, central nervous system involvement by systemic NHL is frequent. Leptomeningeal disease may be present at diagnosis and is asymptomatic in up to $20 \%$ patients with AIDS related systemic $\mathrm{NHL}^{50}$; this is why all patients should have a diagnostic staging lumbar puncture. In addition, the cerebrospinal fluid is a common site of relapse and prophylactic intrathecal chemotherapy should 
be administered to patients considered to be at high risk.

In general, patients with AIDS associated Burkitt's lymphoma are younger and have higher CD 4 cell counts than those who develop diffuse large cell/immunoblastic NHL. ${ }^{41}$ Moreover, Burkitt's lymphoma more often involves the bone marrow and lymph nodes, while the diffuse large cell/immunoblastic tumours more frequently present with extranodal disease involving the gastrointestinal tract and of course include all the primary cerebral lymphomas. ${ }^{51}{ }^{52}$ There is no significant difference in outcome between the two histological groups in most studies.

\section{Prognostic factors}

Early series reported that the most influential prognostic variables for survival related to the severity of immunosuppression rather than lymphoma related factors. This may have been because the majority of patients present with advanced stage, B symptoms and/or extranodal disease and these factors are therefore less discriminatory regarding prognosis. Multivariate analysis of two series revealed that the CD4 count at diagnosis, a history of a previous AIDS defining diagnosis, a poor performance status, and bone marrow involvement were independent adverse prognostic factors. ${ }^{53}{ }^{54} \mathrm{An}$ international prognostic index for lymphoma has been introduced for aggressive lymphomas in the non-HIV infected population ${ }^{55}$ and this scoring system has also been evaluated in HIV associated lymphomas. Elevated serum $\mathrm{LDH}$, age over 40 years, and CD4 lymphocyte count $<100 \times 10^{6} / 1$ were confirmed as adverse prognostic variables. However, more recent prospective data, such as that provided by a recent AIDS Clinical Trials Group (ACTG) study suggested that the prognostic variables in AIDS related lymphoma closely resemble those in the international prognostic index for non-HIV associated non-Hodgkin's lymphoma. ${ }^{56}$

In one series of 73 patients with HIV associated lymphoma from Italy a subgroup of 13 patients who achieved a durable complete remission of at least 2 years was identified. This group had higher CD4 lymphocyte counts and better prognostic scores at presentation than the remaining patients. However, there were no differences in the histological subtypes between the long term survivors and the other patients. ${ }^{57}$ This group reported in 1996 that the 2 year survival for patients with a good prognosis treated with chemotherapy was $50 \%$ compared with $24 \%$ for those with a poor prognosis. ${ }^{58}$ The patients with a good prognosis in complete remission following chemotherapy had a median survival of 34.5 months. In an earlier series published in 1991, a similar group of patients was found to have a median survival of 18 months. ${ }^{54}$ The improvement in median survival in this group of patients probably is real and represents advances in the management of HIV since many of these patients will succumb to HIV related illness rather than the NHL.

\section{Treatment}

Combination chemotherapy is the optimal treatment modality for AIDS associated NHL as patients usually have disseminated disease and extranodal involvement at presentation. Unfortunately, it was recognised early in the epidemic that most patients did not tolerate standard lymphoma chemotherapy well owing to the immunosuppression from HIV and for many patients with adverse prognostic factors the life expectancy was very short. This led clinicians to adopt a prognosis stratified approach to the management of HIV associated NHL.

Patients with the major adverse prognostic factors (low CD4 cell count, poor performance status, and previous AIDS defining illnesses) are treated with palliative intent using mild chemotherapy and/or radiotherapy. The palliative chemotherapy schedules include a combination of oral prednisolone and intravenous vincristine often with intravenous bleomycin. Radiotherapy can be used for localised symptomatic lesions. The life expectancy for these patients is 3-6 months, although there have been no studies that address the role of this palliative chemotherapy approach.

Initially, conventional doses of chemotherapy were used for patients with better prognostic factors. However, marked toxicity and an increased incidence of opportunistic infections led to modifications of the standard lymphoma regimens, such as the modified mBACOD schedule used by the AIDS Clinical Trial Group (ACTG). ${ }^{50}$ Subsequently, the availability of haematopoietic growth factors allowed more myelotoxic schedules to be studied. Full dose mBACOD with granulocytemacrophage colony stimulating factor (GMCSF) support was compared with low dose $\mathrm{mBACOD}$ in a randomised comparison. There was no difference in either response or survival duration; however, an increased incidence of neutropenic sepsis was recorded in the full dose $\operatorname{arm}^{59}$

A number of prospective trials of chemotherapy for HIV associated NHL have been performed based on schedules used in the immunocompetent population which have shown response rates of $33-63 \%$ and median survivals of 5-11 months..$^{53}{ }^{50-62}$ These studies are not directly comparable owing to variations in case mix between series and differences in patient selection. None the less, the response rates and survival data for these trials remain worse than those for HIV negative patients with high grade NHL.

Up to $30-40 \%$ of the patients with good prognostic features will remain in remission following treatment until another AIDS related illness ensues. Recent studies have reported higher median survivals with no change in response rates for AIDS related NHL. ${ }^{63}$ It is speculated that this improvement is due to a reduced incidence of opportunistic infections in the HAART era among patients who have durable remissions of their NHL.

Infusional chemotherapy for AIDS related NHL has been pioneered by Sparano et al using the combination of cyclophosphamide, doxorubicin, and etoposide (CDE) adminis- 
tered as a 96 hour continuous infusion for up to six courses at 4 weekly intervals together with granulocyte colony stimulating factor (G-CSF) and nucleoside reverse transcriptase inhibitors. Initial reports in a selected group of 25 patients with a median CD4 count of $117 \times 10^{6} / 1$ produced an impressive median survival of 18.4 months. ${ }^{64}$ More recently this schedule has been used in conjunction with protease inhibitor based HAART therapy with similar results although there was more mucositis with the protease inhibitor. ${ }^{65}$ Whether the impressive survival with CDE reflects a schedule which truly produces more durable responses on account of the steroid sparing or infusional schedule, or is due to recent improvements in the overall management of HIV disease, remains to be seen. Indeed the preliminary results with CDE from the multicentre Eastern Cooperative Oncology Group trial have been far less impressive ${ }^{66}$ as is so often the case following encouraging initial single centre studies.

HAEMATOPOIETIC GROWTH FACTORS

Patients in the good prognostic category may be treated with conventional NHL chemotherapy regimens with the aim of cure. However, because of the underlying immunodeficiency, poor bone marrow reserve owing to HIV myelodysplasia, and concomitant use of myelosuppressive agents, such as zidovudine and ganciclovir, many patients develop opportunistic infections, neutropenic sepsis, or persisting neutropenia causing chemotherapy delays and hence suboptimal treatment. The use of bone marrow stimulatory factors may facilitate the use of chemotherapy but improved survival has not been demonstrated.

\section{ANTIRETROVIRAL DRUGS}

The concomitant use of antiretroviral agents with chemotherapy is generally acceptable practice with the exception of zidovudine, which significantly adds to the myelosuppression of combination chemotherapy, and didanosine, which may worsen the peripheral neuropathy caused by vinca alkaloids. Little is known about the interactions of protease inhibitors and chemotherapy although the inhibition of p450 may reduce hepatic metabolism of cyclophosphamide and the anthracyclines. For these reasons the infusional schedule EPOCH (etoposide, prednisolone, vincristine, cyclophosphamide, doxorubicin) has been developed at the National Cancer Institute and omits all antiretroviral therapy for the duration of the chemotherapy. In addition to the impressive response rate reported, there has been a restoration of immune function as measured by CD 4 cell count and HIV mRNA viral load within 3 months of the reintroduction of antiretroviral therapy after completing the chemotherapy. ${ }^{67}$

INTRATHECAL CHEMOTHERAPY

There is a high rate of meningeal involvement in HIV associated systemic NHL and it is not necessarily associated with bone marrow involvement or a poor prognosis. Although the frequency of meningeal relapse can be reduced by the use of prophylactic intrathecal chemotherapy this necessitates repeated lumbar punctures. Intrathecal chemotherapy (methotrexate or cytosine arabinoside) should therefore be given to patients with meningeal disease or at high risk of cranial disease by virtue of Burkitt's histology or extensive paranasal sinus and base of skull disease. Most centres also recommend prophylactic intrathecal chemotherapy for patients with bone marrow involvement.

NEW DEVELOPMENTS

MGBG (methyl-glyoxal-bis guanylhydrazone) is a relatively non-myelotoxic spermidine analogue which inhibits cellular polyamine synthesis and has been used with some success in both relapsed systemic NHL and in primary cerebral NHL ${ }^{68}$; however, it has not been licensed. Monoclonal antibodies to IL-6 have been evaluated in AIDS related lymphoma with limited success. ${ }^{69}$ In addition, antibody conjugates have been tested in refractory HIV associated lymphoma. This conjugate was formed of ricin bound to a mouse derived IgG1 monoclonal antibody B1 (antiCD19). CD19 is expressed on normal and malignant B lymphocytes. No significant toxicity was reported in a phase I dose escalating study in nine patients and the response rate was $22 \%{ }^{70}$ Following initial positive results the ricin blocked anti-CD19 antibody conjugate was used in combination with low dose M-BACOD chemotherapy. The complete remission rate was $57 \%$ although the contribution of the immunoconjugate cannot be separated from that of the chemotherapy. ${ }^{71}$ Furthermore, studies of rituximab, a humanised monoclonal antibody to CD20 are under way in this group of patients. ${ }^{72}$

\section{Conclusion}

High grade non-Hodgkin's lymphoma continues to be the second most common neoplasm in people with HIV. The incidence has not changed with the introduction of highly active antiretroviral therapy for HIV despite improved data for other opportunistic cancers, particularly Kaposi's sarcoma. Modified chemotherapy schedules have been used with limited success compared with the HIV negative population. It is hoped that in the future advances in the understanding of the pathogenesis of HIV associated NHL will become the basis for therapeutic strategies directed at the biology of these tumours.

1 Penn I. Cancers complicating organ transplantation. N Engl f Med 1990;323:1767-8.

2 Ockenga J, Tillmann H, Trautwein C, et al. Hepatitis B and $\mathrm{C}$ in HIV-infected patients. Prevalence and prognostic value. F Hepatol 1997;27:18-24.

3 Ziegler JL, Beckstead JA, Volberding PA, et al. NonHodgkin's lymphoma in 90 homosexual men. Relation to generalized lymphadenopathy and the acquired immunodeficiency syndrome. $N$ Engl F Med 1984;311:565-70.

4 Beral V, Peterman T, Berkelman R, et al. AIDS-associated non-Hodgkin lymphoma. Lancet 1991;337:805-9.

5 CDC Surveillence Summaries. Surveillance for AIDSCDC Surveillence Summaries. Surveillance for AIDS-
defining opportunistic illnesses 1992-1997. MMWR 1999; 48(No SS-2).

6 Goedert J, Cote T, Virgo P, et al. Spectrum of AIDSassociated malignant disorders. Lancet 1998;351:1833-9. 
7 Franchesi S, Dal Maso L, La Vecchia C. Advances in the epidemiology of HIV-associated Non-Hodgkin's 1999;83:481-5.

8 Petruckevitch A, Del Amo J, Phillips AN, et al. Disease progression and survival following specific AIDS-defining conditions: a retrospective cohort study of 2048 HIVinfected persons in London. AIDS 1998;12:1007-13

9 Peters BS, Beck EJ, Coleman DG, et al. Changing disease patterns in patients with AIDS in a referral centre in the 1991;302:203-7.

10 Palella F, Delaney K, Moorman A, et al. Declining morbidity and mortality among patients with advanced human immunodeficiency virus infection. N Engl f Med 1998;338: 853-60.

11 Mocroft A, Vella S, Benfield T, et al. Changing patterns of mortality across Europe in patients infected with HIV-1. EuroSIDA Study Group. Lancet 1998;352:1725-30.

12 Ledergerber B, Telenti A, Egger M, et al. Risk of HIV related Kaposi's sarcoma and non-Hodgkin's lymphoma with potent antiretroviral therapy: prospective cohort study BMF 1999;319:23-4

13 Rabkin C, Testa M, von Roenn J. Kaposi's sarcoma and non-Hodgkin's lymphoma incidence and trends in AIDS Clinical Trial Group study participants. F AIDS 1999;21 (Suppl 1):S31-3.

14 Buchbinder S, Holmberg S, Scheer S, et al. Combination antiretroviral therapy and incidence of AIDS-related malignancy. $\mathcal{F}$ AIDS 1999;21 (Suppl 1):S23-6.

15 Jacobsen L, Yamashita T, Margolick J, et al. Impact of potent antiretroviral therapy on the incidence of Kaposi's sarcoma and non-Hodgkin's lymphoma among HIV-1 infected individuals. I AIDS 1999;21 (Suppl 1):S34-41.

16 Knowles D, Chamulak G, Subar M, et al. Lymphoid neoplasia associated with the acquired immunodeficiency
syndrome (AIDS): The New York University Medical syndrome (AIDS): The New York University Medical Center experien

17 Lowenthal D, Strauss D, Campbell S, et al. AIDS-related lymphoid neoplasia. The Memorial Hospital Experience. Cancer 1988;61:2325-37.

18 Roithmann S, Toledano M, Tourami J, et al. HIV-associated non-Hodgkin's lymphomas: clinical characteristics and outcome. The experience of the French Registry of HIV-associated tumours. Ann Oncol 1991;2:289-95.

19 Carbone A, Dolcetti R, Gloghini A, et al. Immunophenotypic and molecular analyses of acquired immune deficiency syndrome-related and Epstein-Barr virus-associated lymphomas: a comparative study. Hum Pathol 1996;27: 133-46.

20 Raphael M, Audouin J, Tulliez M, et al. Anatomic and histologic distribution of 448 cases of AIDS-related non Hodgkin's lymphoma. Proceedings of the annual meeting of the American Society of Hematology. Blood 1993;82:386a (suppl 1)

21 Ioachim HL, Dorsett B, Cronin W, et al. Acquired immunodeficiency syndrome-associated lymphomas: clinical, pathologic, immunologic, and viral characteristics of 111 cases. Hum Pathol 1991;22:659-73.

22 Cesarman E, Chang Y, Moore PS, et al. Kaposi's sarcoma-associated herpesvirus-like DNA sequences in AIDS-related body-cavity-based lymphomas. $N$ Engl f Med 1995;332:1186-91.

23 Oksenhendler E, Duarte M, Soulier J, et al. Multicentric Castleman's disease in HIV infection: a clinical and pathological study of 20 patients. AIDS 1996;10:61-7.

24 Dupin N, Diss T, Kellam P, et al. HHV-8 is associated with a plasmablastic variant of Castleman disease that is linked to HHV-8-positive plasmablastic lymphoma. Blood 2000; to HHV-8-p $1406-12$.

25 Schnittman S, Lane H, Higgins S, et al. Direct polyclonal activation of human B lymphocytes by the AIDS virus. Science 1986;233:1084-6.

26 Nakajima K, Martinez-Maza O, Hirano T, et al. Induction of IL-6 production by human immunodeficiency virus. $\mathcal{F}$ Immunol 1989;142:531-6.

27 Emilie D, Coumbaras J, Raphael M, et al. Interleukin-6 production in high grade lymphoma: Correlation with the presence of malignant immunoblasts in acquired immunodeficiency syndrome and in human immunodeficiency virus seronegative patients. Blood 1992;80:498-504.

28 Masood R, Zhang Y, Bond M, et al. Interleukin 10 is an autocrine growth factor for AIDS-related B cell lymphoma. Blood 1995;85:3423-30.

29 Pastore C, Gaidano G, Ghia P, et al. Patterns of cytokine expression in AIDS-related non-Hodgkin's lymphoma. $B$ 7 Haematol 1999;103:143-9.

30 ersten M, Van Gorp J, Pals S, et al. Expression of Epstein-Barr virus latent genes and adhesion molecules in AIDS-related non-Hodgkin's lymphoma: correlation with histology and CD4 cell number. Leuk Lymphoma 1998;30 515-24.

31 Soulier J, Grollet L, Oksenhendlder E, et al. Kaposi's sarcoma-associated herpesvirus-like DNA sequences in
multicentric Castleman's disease. Blood 1995;86:1276-80.

32 Chaganti R, Jhanwar S, Koziner B, et al. Specific translocations characterize Burkitt-like lymphoma in homosexual men with the acquired immunodeficiency syndrome. Blood 1983;61:1265-8.

33 Subar M, Neri A, Inghirami G, et al. Frequent c-myc oncogene activation and infrequent presence of Epstein Barr
virus genome in AIDS-associated lymphoma. Blood 1988; virus genom
34 Gaidano G, Carbone A, Dalla-Favera R. Pathogenesis of AIDS-related lymphomas: molecular and histiogenetic AIDS-related lymphomas: molecular and

35 Gaidano G, LoCoco F, Ye B, et al. Rearrangement of the Bcl-6 gene in AIDS-associated non-Hodgkin's lymphoma. Blood 1994;84:397-402.

36 Gaidano G, Carbone A, Pastore C, et al. Frequent mutation of the 5' noncoding region of the bcl-6 gene in acquired immunodeficiency syndrome-related non-Hodgkin's lymphomas. Blood 1997;89:3755-62.

37 Gaidano G, Capello D, Gloghini A, et al. BCL-6 in AIDS-related lymphomas: pathogenetic and histogenetic implications. Leuk Lymphoma 1999;31:39-46.

38 Dean M, Jacobson L, McFarlane G, et al. Reduced risk of AIDS lymphoma in individuals heterozygous for the CCR5-delta32 mutation. Cancer Res 1999;59:3561-4.

39 Rabkin C, Yang Q, Goedert J, et al. Chemokine and chemokine receptor gene variants and risk of non-Hodgkin's lymphoma in human immunodeficiency virus 1 infected individuals. Blood 1999;93:1838-42.

40 Liu R, Paxton W, Choe S, et al. Homozygous defect in HIV-1 co-receptor accounts for resistance of some multiply exposed individuals to HIV-1 infection. Cell 1996;86:367-

41 Samson M, Libert F, Doranz B, et al. Resistance to HIV-1 nfection in caucasian individuals bearing mutnant allele of the CCR-5 chemokine receptor gene. Nature 1996;382: $722-5$.

42 Dean M, Carrington M, Winkler C, et al. Genetic restriction of HIV-1 infection and progression to AIDS by a deletion of the CKR5 structural gene. Science 1996;273:1856-62.

$43 \mathrm{Ng} \mathrm{V}$, Fein C, Knayam-Bashi F, et al. Immunoglobulin secreted by an AIDS lymphoma cell line recognises HIV gp160, suggesting that the immune response to HIV antigens may contribute to lymphomagenesis. Int Conf AIDS 1990;6:202.

44 Herndier B, Shiramizu B, Jewett N, et al. Acquired immunodeficiency syndrome-associated T-cell lymphoma: evidence for human immunodeficiency virus type
1-associated T-cell transformation. Blood 1992;79:176874 .

45 Obrams G, Gruffernan S. Epidemiology of HIV associated NHL. Cancer Surv 1991;10:91-102.

46 Levine A. Acquired immunodeficiency syndrome-related lymphoma. Blood 1992;80:8-20.

47 Ioachim H. Lymphoma: an opportunistic neoplasm of AIDS. Leukemia 1992;6(Suppl 3):65-95.

48 Benmiloud M, Benabed K, Ben Othman T, et al. Results using chemotherapy in AIDS patients with hepatic non-Hodgkin's lymphoma (HNHL). Proc Am Soc Clin Oncol 1999;18:12a.

49 Seneviratne L, Tupule A, Mumannemi M, et al. Clinical immunological and pathological correlates of bone marrow involvement in 253 patients with AIDS-related lymphoma. Blood 1998;92:244a.

50 Levine AM, Wernz JC, Kaplan L, et al. Low dose chemotherapy with central nervous system prophylaxis and zidovudine maintenance in AIDS-related lymphoma. FAMA 1991;266:84-8

51 Roithmann S, Tourani J, Andrieu J. AIDS-associated non-Hodgkin's lymphoma. Lancet 1991;338:884.

52 MacMahon E, Glass J, Hayward S, et al. Epstein-Barr virus in AIDS-related primary central nervous system lymphoma. Lancet 1991;338:969-73.

53 Kaplan LD, Abrams DI, Feigal E, et al. AIDS-associated non-Hodgkin's lymphoma in San Francisco. ҒAMA 1989; 261:719-24

54 Levine AM, Sullivan Halley J, Pike MC, et al. Human immunodeficiency virus-related lymphoma. Prognostic factors predictive of survival. Cancer 1991;68:2466-72.

55 The International Non-Hodgkin's Lymphoma Prognostic Factors Project. A predictive model for aggressive nonHodgkin's lymphoma. N Engl f Med 1993;329:987-94.

56 Straus DJ, Huang J, Testa MA, et al. Prognostic factors in the treatment of human immunodeficiency virusassociated non-Hodgkin's lymphoma: analysis of AIDS Clinical Trials Group protocol 142-low-dose versus standard-dose m-BACOD plus granulocyte-macrophage colony-stimulating factor. National Institute of Allergy and Infectious Diseases. F Clin Oncol 1998;16:3601-6.

57 Tirelli U, Errante D, Spina M, et al. Long-term survival of patients with HIV-related systemic non-Hodgkin's lymphomas. Hematol Oncol 1996;14:7-15.

58 Vaccher E, Tirelli U, Spina M, et al. Age and serum lactate dehydrogenase level are independent prognostic factors in human immunodeficiency virus-related non-Hodgkin's lymphomas: a single-institute study of 96 patients. $\mathcal{F}$ Clin Oncol 1996;14:2217-23.

59 Kaplan L, Straus D, Testa M, et al. Randomized trial of standard dose mBACOD with GM-CSF vs reduced dose $\mathrm{mBACOD}$ for systemic HIV-associated lymphoma: ACTG 142. Proc Am Soc Clin Oncol 1995;14:288.

60 Kaplan LD, Straus DJ, Testa MA, et al. Low dose compared with standard dose m-BACOD chemotherapy for nonHodgkin's lymphoma associated with human immunodeficiency virus infection. $N$ Engl f Med 1997;336:1641-8.

61 Gisselbrecht C, Oksenhendler E, Tirelli U, et al. Human immunodeficiency virus-related lymphoma treatment with intensive combination chemotherapy. French-Italian Cooperative Group. Am f Med 1993;95:188-96.

62 Remick S, McSharry J, Wolf B, et al. Novel oral combination chemotherapy in the treatment of intermediate grade and high grade AIDS-related non-Hodgkin's lymphoma. $f$ Clin Oncol 1992;11:1691-1702. 
63 Bower M, Stern S, Fife K, et al. Weekly alternating combination chemotherapy for good prognosis AIDS combination chemotherapy for good progno
related lymphoma. Eur 7 Cancer 2000 ; (in press).

64 Sparano JA, Wiernik PH, Hu X, et al. Pilot trial of infusional cyclophosphamide, doxorubicin and etoposide plus didanosine and filgrastim in patients with HIV associated non-Hodgkin's lymphoma. f Clin Oncol 1996;14:302635.

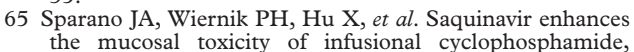
doxorubicin and etoposide in patients with HIVassociated non-Hodgkin's lymphoma. Med Oncol 1998;15: $50-7$.

66 Sparano J, Lee S, Chen M, et al. Phase II trial of infusional cyclophosphamide, doxorubicin and etoposide (CDE) in HIV-associated non-Hodgkin's lymphoma: An Eastern Cooperative Oncology Group trial (E1494). Proc Am Soc Clin Oncol 1999;18:12a.

67 Little R, Pearson D, Steinberg S, et al. Dose-adjusted EPOCH chemotherapy in previously untreated HIV- associated non-Hodgkin's lymphoma. Proc Am Soc Clin

Oncol 1999;18:10a.
68 Levine A, Tulpule A, Espina B, et al. Mitoguazone (MGBG) with radiation therapy in AIDS-related primary CNSlymphoma. Proceedings of the XI International Conference on AIDS 1996;2:222.

69 Emilie D, Marfaing A, Merrien D, et al. Treatment of AIDS-lymphomas with an anti-IL-6 monoclonal antibody. Blood 1993;82:387a

70 Tulpule A, Anderson LJJ, Levine AM, et al. Anti-B4 (CD19) monoclonal antibody conjugated with ricin (B4-blocked ricin) in refractory AIDS-lymphoma. Proc Am Soc Clin Oncol 1994;13:52.

71 Scadden D, Schenkein D, Bernstein Z, et al. Immunotoxin combined with chemotherapy for patients with AIDSrelated non-Hodgkin's lymphoma. Cancer 1998;83:25807.

72 Barrett J, Linn C, Saleh M. Rituximab for the treatment of AIDS-associated non-Hodgkin's lymphoma. F AIDS 1999; 21:A40. 